\title{
EDITORIAL AGING IN EUROPE
}

\author{
Prof. Dr. Jean-Pierre BAEYENS
}

President IAGG-European Region

In 1950 some very clever scientists met in Liège (Belgium) on the invitation of Prof. Brull (specialist in Internal medicine) and launched the International Association of Gerontology (IAG). By-laws were made and registered as charity in Belgium. The scientists were from different origins: clinicians, biologists, sociologists, demographers, economists etc. They were convinced that Gerontology could only develop in a multidisciplinary context.

We are now 60 years later. We see Gerontology developing in many different societies and little groups. Every subdiscipline is doing Gerontology on (his) its own. That is in fact not a good evolution.

It is necessary for the IAG, now IAGG, to go back to (his) its roots: all research has to be imbedded in a multidisciplinary approach. The IAGG has to find a way to be an umbrella organisation for all these (organisations) set-ups and groups, as it was in the beginning.

In September 2008 the IAGG-European Region participated in a multidisciplinary and international EU meeting in Wroclaw (in) for the (redaction) writing of the "Silver Paper", published in the JNHA.

With a World Congress and Regional Congress every four years, we are loosing after each (congress) event, the contact with national societies and their members, with delegates, with pharmaceutical companies and with media. The European Region is therefore advocating to change by-laws and to organize World Congresses every two years, alternatively with a European Congress every other two years. This (will) should increase visibility of Gerontology in the world.

The European Region is also very concerned with the foreseen launching of the African Region. Indeed, historically, Africa (and especially North Africa) had, and still has, many links with Europe. For more than 3000 years the "Mediterranean Region" is in fact a reality.

For all these aspects of IAGG, the next Paris World Congress will be a major issue! 